\title{
Therapeutic effects of soluble dietary fiber consumption on type 2 diabetes mellitus
}

\author{
CHUNYE CHEN $^{1 *}$, YUAN ZENG $^{1 *}$, JING XU $^{2}$, HONGTING ZHENG ${ }^{2}$, JUN LIU $^{3}$, RONG FAN $^{3}$, \\ WENYI ZHU ${ }^{3}$, LIJIA YUAN ${ }^{1}$, YU QIN ${ }^{1}$, SHIHUI CHEN ${ }^{1}$, YONG ZHOU ${ }^{1}$, YING WU ${ }^{1}$, \\ JING WAN $^{1}$, MANTIAN MI ${ }^{1}$ and JIAN WANG ${ }^{3}$ \\ ${ }^{1}$ Research Center of Nutrition and Food Safety, Chongqing Key Laboratory of Nutrition and Food Safety; \\ Departments of ${ }^{2}$ Endocrinology and ${ }^{3}$ Nutrition, Xinqiao Hospital, The Third Military Medical University, \\ Shapingba, Chongqing 400038, P.R. China
}

Received February 21, 2015; Accepted April 1, 2016

DOI: $10.3892 /$ etm.2016.3377

\begin{abstract}
Soluble dietary fiber (DF) reduces the risk of developing diabetes and may have therapeutic effects in patients with type 2 diabetes mellitus (DM2). The present study aimed to investigate the effect of soluble DF on metabolic control in patients with DM2. A total of 117 patients with DM2 between the ages of 40 and 70 were assessed. Patients were randomly assigned to one of two groups, and administered extra soluble DF (10 or $20 \mathrm{~g} / \mathrm{day})$, or to a control group ( $0 \mathrm{~g} / \mathrm{day})$ for one month. Blood glucose, serum insulin and connecting peptide (C-peptide) levels, and the insulin resistance index, as determined using the homeostatic model assessment method, were measured during fasting and up to 2-h postprandially prior to and following one month of treatment. Other measurements included serum levels of glycated albumin (GA), blood lipid profiles, and an analysis of the blood pressure, body weight and waist/hip ratio of all patients. Following intervention, the levels of 2-h blood glucose, fasting insulin and lipoprotein(a), and the insulin resistance index, were significantly improved in all groups. Furthermore, the fasting blood glucose, 2-h insulin, fasting C-peptide, 2-h C-peptide, GA and triglyceride (TG) levels were significantly improved in the soluble DF
\end{abstract}

Correspondence to: Professor Mantian Mi, Research Center of Nutrition and Food Safety, Chongqing Key Laboratory of Nutrition and Food Safety, The Third Military Medical University, 30 Gaotanyan Street, Shapingba, Chongqing 400038, P.R. China E-mail:mimantiancn@126.com

Professor Jian Wang, Department of Nutrition, Xinqiao Hospital, The Third Military Medical University, 183 Xinqiao Street, Shapingba, Chongqing 400038, P.R. China

E-mail: wangjiannd@yeah.net

*Contributed equally

Key words: type 2 diabetes mellitus, soluble dietary fiber, blood glucose, insulin resistance index, glycated albumin groups. The $20 \mathrm{~g} /$ day soluble DF group exhibited significantly improved fasting blood glucose and low-density lipoprotein levels, as well as a significantly improved insulin resistance index. In addition, 10 and $20 \mathrm{~g}$ /day soluble DF significantly improved the waist and hip circumferences and levels of TGs and apolipoprotein A. The results of the present study suggested that increased and regular consumption of soluble DF led to significant improvements in blood glucose levels, insulin resistance and metabolic profiles, without improving the secretory function of the islets of Langerhans, over a short-term intervention period in patients with DM2.

\section{Introduction}

Type 2 diabetes mellitus (DM2) is associated with numerous complications, including kidney failure, blindness, an increased susceptibility to infection, coronary heart disease and stroke (1). It is predicted that by $2030 \sim 10 \%$ of the world's population will have diabetes mellitus (DM) (mostly type 2) (2). Interventions that improve diet quality have been demonstrated to be effective in controlling hyperglycemia and its associated risk factors, which in turn reduces the risk of diabetes-associated complications (3). Considering the seriousness of DM2 complications, early dietary education is critical for delaying or preventing disease onset. Accordingly, the American Diabetes Association (ADA) has recommended dietary guidelines for patients with DM2 (4).

Dietary fiber (DF) is widely prescribed (5), either alone or in combination with lipid-lowering therapies, to reduce cholesterol levels (6). The exact mechanism by which soluble fiber lowers serum levels of low-density lipoprotein (LDL) and cholesterol is not completely understood; however, it has been suggested that soluble fiber may interfere with lipid and/or bile acid metabolism (7). A reasonable increase in DF intake (20-35 g/day) is recommended by the ADA based on the effects of soluble fiber on plasma levels of cholesterol (4). Recent epidemiological findings have suggested that there is an association between high DF intake and a reduced risk of developing diabetes and coronary heart disease $(8,9)$. In particular, soluble DF has been shown to reduce insulin resistance in female non-diabetic patients (10). However, there 
remains a lack of clinical intervention studies with sufficient sample sizes to support the beneficial effects of DF intake in patients with DM2.

The present study aimed to investigate the effects of DF on glycemic control and plasma lipid concentrations in patients with DM2. Patients were administered DF at doses greater than the ADA-recommended level of 25-35 $\mathrm{g}$ in order to elucidate how a high-fiber diet may affect plasma cholesterol levels, intestinal cholesterol absorption and fecal sterol excretion (11).

\section{Materials and methods}

Patients. A total of 120 patients at the Xinqiao Hospital of the Third Military Medical University (Chongqing, China), who had been diagnosed with DM2 [based on the 2010 ADA criteria (12)] for $\geq 6$ months, were enrolled in the present study between December 2012 and February 2013 (clinical trial registration no. ChiCTR-TRC-12002580). The majority of the enrolled patients had been diagnosed after reaching $\geq 40$ years of age. The age of the patients ranged from 45-70 years (average age, $54 \pm 9$ years), and they had a mean body weight of $68.1 \pm 13.0 \mathrm{~kg}$ and a mean body mass index (BMI) of $25.3 \pm 3.9$ $\mathrm{kg} / \mathrm{m}^{2}$. A total of 40 patients were treated with the ADA diet alone, whereas the remaining 80 patients were treated with 10 or $20 \mathrm{mg}$ soluble DF daily in addition to the ADA diet for one month. Upon study entry, all patients were free of tumors, myocardial infarction, unstable angina pectoris and congestive heart failure. Furthermore, no thyroid or hepatic disease was found, and none of the patients were undergoing lipid-lowering treatments.

Written informed consent was obtained from all patients prior to participation. The present study was approved by the Ethics Committee of the Third Military Medical University. All procedures were conducted in accordance with the Declaration of Helsinki.

Study design. The present study was a randomized, double-blind trial. The three common treatments for patients with DM2 include oral medication, insulin therapy or a combination of both. Subjects that met the inclusion criteria were stratified according to treatment and randomly divided into the following three groups using a random number table (40 subjects/group): i) Control; ii) low-dose (10DF); and iii) high-dose (20DF) groups. All patients received medical nutrition therapy (MNT), which include personal nutritional assessment and reasonable nutritional intervention. Lifestyle interventions consisted of individualized dietary counseling and encouragements to increase daily physical activity. Each patient received an individualized recommended intake of daily energy according to the ADA guidelines (12). After one month of treatment, 37 patients remained in the control group, 3 patients quit for personal reasons and 40 patients remained in the $10 \mathrm{DF}$ and $20 \mathrm{DF}$ groups, respectively. In the control group, 16 patients received oral medicine [ $500 \mathrm{mg}$ of metformin hydrochloride tablets (Bristol-Myers Squibb Company, Princeton, NJ, USA) twice a day and $50 \mathrm{mg}$ of acarbose tablets (Bayer HealthCare Pharmaceuticals Inc., Leverkusen, Germany) three times daily], 9 patients self-administered insulin injections and 12 patients took both. In the 10DF group, 17 patients received oral medicine [(500 $\mathrm{mg}$ of metformin hydrochloride tablets twice a day and $50 \mathrm{mg}$ of acarbose tablets three times daily)], 10 patients self-administered insulin injections and 13 patients took both. In the 20DF group, 17 patients received oral medicine $(500 \mathrm{mg}$ of metformin hydrochloride tablets twice a day and $50 \mathrm{mg}$ of acarbose tablets three times daily), 11 patients self-administered insulin injections and 12 patients took both. The constituent ratios of medication among the three groups were not significantly different $(\mathrm{P}=0.894)$. Patients in the control group received MNT only, and patients in the 10DF and 20DF groups were provided with 10 and $20 \mathrm{~g}$ soluble DF each day, respectively. The total duration of the trial was one month, during which time the subjects received MNT treatment. All participants received a weekly consultation, during which the patient's protocol compliance was assessed For patients with a poor compliance, more frequent calls were made to ensure that they took enough DF. The body weight, blood pressure and waist and hip circumferences of all patients were measured prior to and following treatment by clinical lab technicians who were blinded to the content and purpose of the study. In addition, the patients were asked to complete a 3-day record of their food intake at the baseline and end of the trial, which were analyzed using NCCW 2011 software (Dongcheng New technology Co., Ltd., Qingdao, China) to estimate nutrient intake. Furthermore, the patients were asked to complete a questionnaire prior to treatment regarding their dietary habits, including how frequently they consumed meat, milk, eggs, vegetables and other foods, and if they regularly consumed vitamin or mineral supplements.

Subjects reported a normal bowel habit and denied any symptoms of difficult defecation or rectal bleeding on a symptom questionnaire that inquired about stool frequency, straining effort, feeling of incomplete evacuation, use of digital maneuvers, painful defecation, hard stools, abdominal pain, and reflux symptoms (13).

Biochemical analyses. Blood samples $(12 \mathrm{ml})$ were collected in anticoagulant-free tubes [for serum lipids, insulin, connecting peptide (C-peptide), urea nitrogen, and creatinine assays; Zhongshan Jinqiao Biotechnology Co., Ltd., Beijing, China] and fluoride-oxalate anticoagulant tubes (for the plasma glucose assay; Zhongshan Jinqiao Biotechnology Co., Ltd.). Anticoagulant-free tubes (Zhongshan Jinqiao Biotech Co.,Ltd.) were allowed to clot in the dark at room temperature for $2 \mathrm{~h}$ and held for $2 \mathrm{~h}$, and the fluoride-oxalate tubes were immediately placed on ice. Both tubes were centrifuged at $1,000 \mathrm{x} \mathrm{g}$ for $20 \mathrm{~min}$ at $4^{\circ} \mathrm{C}$. Serum and plasma were separated and frozen at $-80^{\circ} \mathrm{C}$ until further analysis. All biochemical analyses on samples from individual participants were performed together to eliminate inter-assay variability. Plasma glucose, serum lipid and lipoprotein(a) (Lpa) levels were analyzed using a blood biochemical analyzer (AU2700; Olympus Corporation, Tokyo, Japan). Plasma glucose, serum total cholesterol, high-density lipoprotein (HDL) and triglycerides (TG) were measured using enzymatic reagents (Zhongshan Jinqiao Biotechnology Co., Ltd.). LDL (TA309729), apolipoprotein (apo; TA318199), serum insulin (TA306175), serum glycated albumin (GA; TA307256), C-peptide (TA317763), urea nitrogen (TA306427), and creatinine levels (TA305574) were measured by radioimmunoassays using commercially available kits (Chongqing Keyuan Medical Equipment Co., Ltd., 


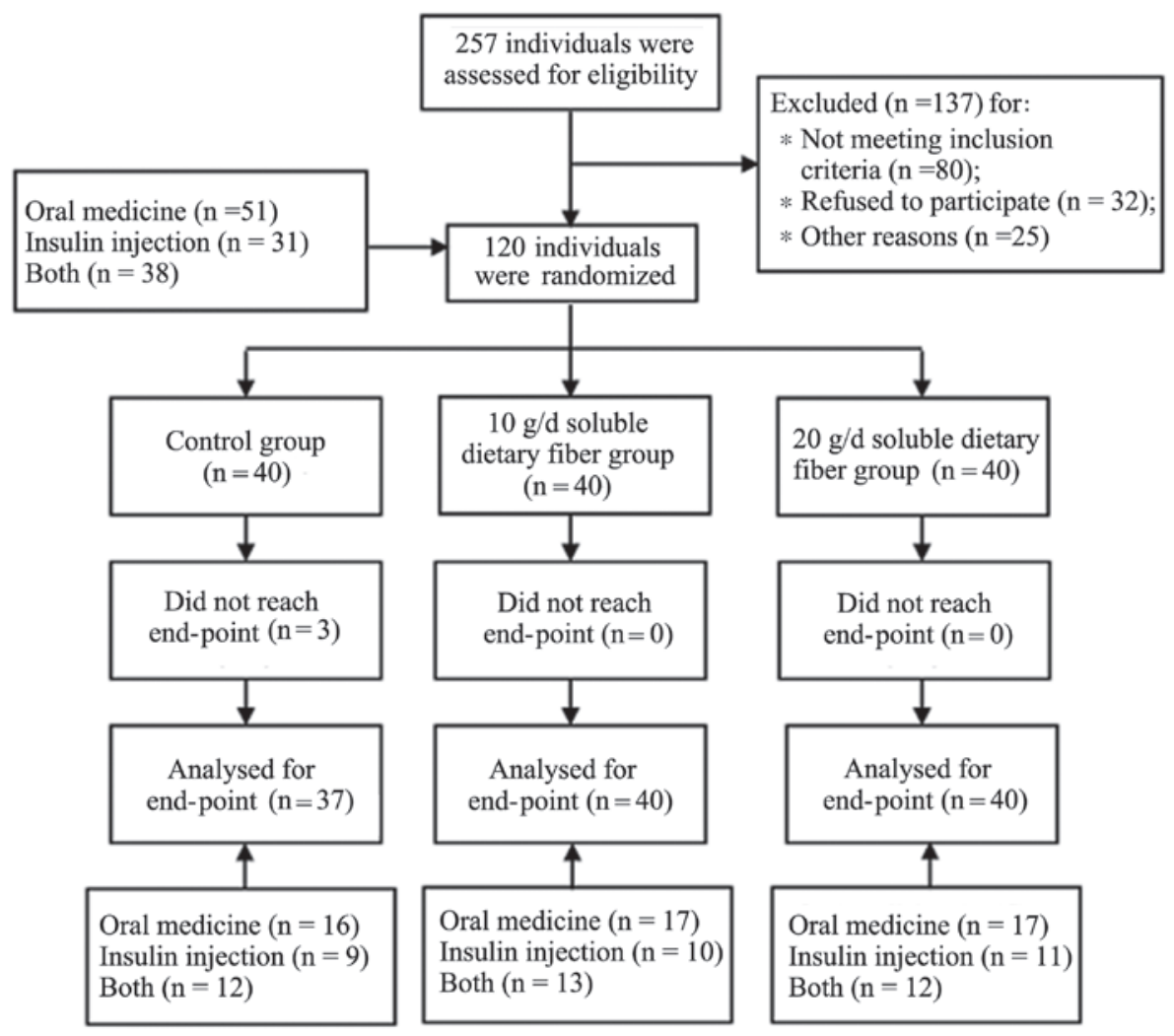

Figure 1. Flow diagram of participants.

Chongqing, China). The intra-assay coefficients of variance for serum lipids, lipoproteins and plasma glucose were $<2 \%$ and $<6 \%$ for serum insulin and C-peptide, respectively. The insulin resistance index was determined using the homeostatic model assessment method (14). Insulin resistance and $\beta$-cell function were estimated from fasting glucose and insulin levels.

Statistical analysis. Data were analyzed using SPSS software, version 19.0 (IBM SPSS, Armonk, NY, USA). Data are presented as the mean \pm standard deviation. $95 \%$ confidence intervals (CIs) were calculated for the mean values between groups. The Shapiro-Wilk test demonstrated that all of the main outcome variables were normally distributed. Differences in patient characteristics between pre- and post-treatments were assessed using paired Student's t-tests. One-way analysis of covariance, with the difference between pre- and post-treatment set as the dependent variable and the baseline value as the covariant, was used to compare the soluble DF groups with the control group for blood markers and anthropometric characteristics. $\mathrm{P}<0.05$ was considered to indicate a statistically significant difference.

\section{Results}

Participant flow. Based on patient interviews, estimates of dietary energy content and the remainder of soluble DF, a satisfactory level of patient compliance was achieved in the present study. A participant flow diagram is shown in Fig. 1.

Baseline data and participant characteristics. According to the baseline energy intake questionnaire survey, the 20DF group consumed significantly more Vitamin $\mathrm{C}$, as compared with the other groups $(\mathrm{P}<0.05)$. However, no other significant differences in dietary intake were observed between the groups. The mean energy intake of all groups was $2302.78 \pm 872.48 \mathrm{kcal} /$ day. The average percentage of calories obtained from fat intake was $36.35 \%$, which was markedly higher than the ADA recommendation (20-30\%). The average intake of DF was $15.91 \mathrm{~g}$, which was markedly lower than the ADA recommendation (25-35 g) (Table I). The age, BMI, waist/hip ratio, blood glucose and insulin levels, and several other baseline characteristics, were recorded for all patients. No significant differences were observed between groups for the baseline characteristics (Table II).

Outcomes and estimation. According to the energy intake questionnaire survey completed one month post-intervention, the $10 \mathrm{DF}$ and the $20 \mathrm{DF}$ groups consumed significantly more $\mathrm{DF}$, as compared with the control group $(\mathrm{P}<0.01)$. Energy, protein, fat, carbohydrate and cholesterol intakes were significantly lower in the energy intake questionnaires completed one month post-intervention, as compared with the baseline questionnaires $(\mathrm{P}<0.05)$. Furthermore, the calories from fat and carbohydrate intake were significantly decreased in the one month post-intervention questionnaire, as compared with the baseline questionnaire $(\mathrm{P}<0.05$; Table I). The results of the blood marker analysis, including anthropometric measures, lipid profile and DM2 biomarkers, and assessment of physical characteristics, at one month following intervention are summarized in Table III. After one month of treatment, the systolic pressure and levels of HDL, LDL and apoB were significantly improved in the 20DF group as compared with the 


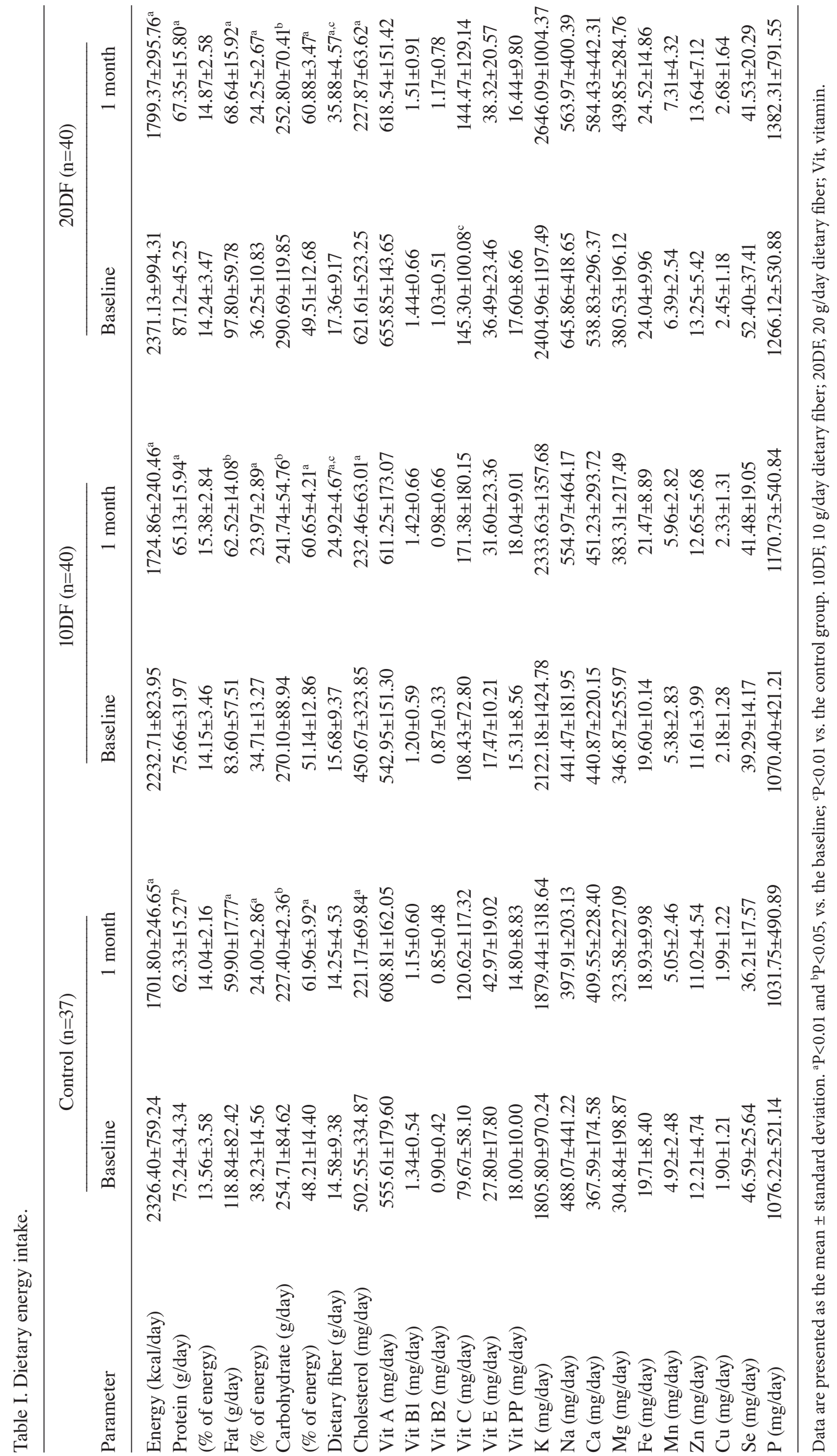


Table II. Baseline characteristics.

\begin{tabular}{|c|c|c|c|c|c|}
\hline Parameter & Control & $10 \mathrm{DF}$ & $20 \mathrm{DF}$ & F-value & P-value \\
\hline $\mathrm{n}$ & 37 & 40 & 40 & & \\
\hline Age & $51.52 \pm 6.47$ & $53.63 \pm 9.37$ & $55.52 \pm 8.15$ & & \\
\hline Gender (M/F) & $14 / 23$ & $15 / 25$ & $17 / 23$ & & \\
\hline Weight (kg) & $69.60 \pm 8.50$ & $65.30 \pm 10.20$ & $69.50 \pm 13.00$ & 2.066 & 0.131 \\
\hline $\operatorname{BMI}\left(\mathrm{kg} / \mathrm{m}^{2}\right)$ & $25.65 \pm 2.62$ & $24.80 \pm 2.92$ & $25.32 \pm 3.96$ & 0.675 & 0.511 \\
\hline Waist circumference $(\mathrm{cm})$ & $90.50 \pm 8.10$ & $90.70 \pm 9.79$ & $91.31 \pm 10.90$ & 0.071 & 0.931 \\
\hline Hip circumference $(\mathrm{cm})$ & $100.90 \pm 7.71$ & $100.00 \pm 7.01$ & $100.61 \pm 7.21$ & 0.144 & 0.866 \\
\hline Waist/hip ratio & $0.90 \pm 0.06$ & $0.91 \pm 0.06$ & $0.91 \pm 0.06$ & 0.239 & 0.788 \\
\hline Systolic pressure (mmHg) & $128.46 \pm 15.87$ & $132.85 \pm 17.29$ & $130.75 \pm 15.53$ & 0.701 & 0.498 \\
\hline Diastolic pressure (mmHg) & $75.54 \pm 10.72$ & $79.58 \pm 12.19$ & $79.15 \pm 10.23$ & 1.517 & 0.224 \\
\hline Fasting blood glucose (mmol/l) & $8.12 \pm 2.78$ & $8.26 \pm 2.84$ & $8.71 \pm 2.91$ & 0.459 & 0.633 \\
\hline 2-h blood glucose $(\mathrm{mmol} / \mathrm{l})$ & $12.55 \pm 4.39$ & $12.50 \pm 4.21$ & $12.54 \pm 3.88$ & 0.002 & 0.998 \\
\hline Fasting insulin (mU/l) & $9.55 \pm 7.06$ & $9.27 \pm 5.75$ & $9.81 \pm 5.60$ & 0.076 & 0.927 \\
\hline 2-h insulin (mU/l) & $23.78 \pm 16.52$ & $24.94 \pm 23.20$ & $24.96 \pm 14.72$ & 0.226 & 0.798 \\
\hline HOMA-IR & $3.34 \pm 2.64$ & $3.40 \pm 2.59$ & $3.92 \pm 2.77$ & 0.549 & 0.579 \\
\hline Fasting C-peptide (ng/ml) & $2.25 \pm 1.03$ & $2.78 \pm 1.57$ & $2.59 \pm 1.39$ & 1.457 & 0.237 \\
\hline 2-h C-peptide (ng/ml) & $5.28 \pm 4.05$ & $6.57 \pm 4.20$ & $6.15 \pm 3.01$ & 0.013 & 0.987 \\
\hline Glycated albumin (\%) & $18.84 \pm 4.74$ & $20.00 \pm 4.31$ & $20.64 \pm 5.35$ & 1.368 & 0.259 \\
\hline Cholesterol (mmol/l) & $4.83 \pm 2.16$ & $4.84 \pm 1.17$ & $5.07 \pm 1.31$ & 0.287 & 0.751 \\
\hline Triglyceride (mmol/l) & $2.08 \pm 1.58$ & $2.68 \pm 2.68$ & $2.40 \pm 2.68$ & 0.599 & 0.551 \\
\hline $\mathrm{HDL}(\mathrm{mmol} / \mathrm{l})$ & $1.12 \pm 0.42$ & $1.27 \pm 0.41$ & $1.36 \pm 0.71$ & 1.983 & 0.142 \\
\hline LDL (mmol/l) & $2.92 \pm 2.71$ & $2.45 \pm 0.74$ & $2.90 \pm 0.80$ & 1.008 & 0.368 \\
\hline Lpa (mg/l) & $292.16 \pm 258.47$ & $311.38 \pm 282.61$ & $313.15 \pm 227.88$ & 0.078 & 0.925 \\
\hline $\operatorname{ApoA}(g / l)$ & $1.12 \pm 0.27$ & $1.25 \pm 0.29$ & $1.29 \pm 0.49$ & 2.294 & 0.106 \\
\hline ApoB (g/l) & $1.42 \pm 0.36$ & $1.30 \pm 0.49$ & $1.52 \pm 0.48$ & 2.007 & 0.132 \\
\hline Urea nitrogen $(\mathrm{mmol} / \mathrm{l})$ & $6.00 \pm 3.68$ & $5.91 \pm 1.73$ & $5.86 \pm 2.01$ & 0.029 & 0.971 \\
\hline Creatinine $(\mu \mathrm{mol} / \mathrm{l})$ & $64.09 \pm 22.49$ & $59.58 \pm 20.60$ & $60.36 \pm 15.28$ & 0.574 & 0.565 \\
\hline Uric acid $(\mu \mathrm{mol} / \mathrm{l})$ & $289.65 \pm 104.52$ & $304.83 \pm 92.57$ & $296.78 \pm 76.57$ & 0.265 & 0.768 \\
\hline
\end{tabular}

Data are presented as the mean \pm standard deviation. 10DF, $10 \mathrm{~g}$ /day dietary fiber; 20DF, $20 \mathrm{~g} /$ day dietary fiber; M, male; F, female; BMI, body mass index; HOMA-IR, homeostatic model assessment-insulin resistance; HDL, high-density lipoprotein; LDL, low-density lipoprotein; Lpa, lipoprotein(a); Apo, apolipoprotein.

data prior to the treatment $(\mathrm{P}<0.05)$. In addition, weight, $\mathrm{BMI}$ and hip circumference values were significantly improved in the control and 10DF groups $(\mathrm{P}<0.05)$, and the waist circumference was significantly reduced in the $10 \mathrm{DF}$ group $(\mathrm{P}<0.05)$ as compared with the data prior to the treatment. Fasting insulin, 2-h blood glucose and Lpa levels, and the insulin resistance index, as determined using the homeostatic model assessment method, were significantly improved in all three groups as compared with the data prior to the treatment $(\mathrm{P}<0.05)$. Furthermore, the fasting blood glucose, 2-h insulin, fasting C-peptide, 2-h C-peptide, GA and TG levels were significantly improved in the DF groups $(\mathrm{P}<0.05)$, as compared with the control group, where no significant improvements for these metrics were observed.

Differences between pre- and post-treatment among the three groups are compared in Table IV. Univariate analysis of general linear models, with the difference between pre- and post-treatment as the dependent variable and the baseline value as the covariant, was used. Levels of fasting blood glucose and LDL, and the insulin resistance index, were significantly improved post-treatment in the 20DF group $(\mathrm{P}<0.05)$, but not in the 10DF group. Both 10 and $20 \mathrm{~g} /$ day soluble DF significantly improved the 2 -h blood glucose and GA levels $(\mathrm{P}<0.05)$, although $20 \mathrm{~g} /$ day soluble DF had a greater effect $(\mathrm{P}<0.05)$. Furthermore, both 10 and $20 \mathrm{~g} / \mathrm{d}$ soluble DF significantly improved the waist and hip circumferences and TG and apoA levels $(\mathrm{P}<0.05)$, and no significant differences were found between the 10DF and 20DF groups for these parameters. No significant differences between the 10DF and 20DF were observed for weight, BMI, blood pressure and levels of fasting insulin, 2-h insulin, fasting C-peptide, 2-h C-peptide, cholesterol, HDL, Lpa, apoB, urea nitrogen, creatinine and uric acid.

Safety data. To assess the safety of increased soluble DF intake, the renal function of all patients was measured. No significant differences were observed between the baseline and one month post-intervention measurements for all three groups (Table V). Seven patients in the 20DF group and two 
Table III. Anthropometric measures, lipid profile and type 2 diabetes mellitus biomarkers after 1 month of treatment.

\begin{tabular}{|c|c|c|c|c|c|}
\hline Parameter & Group & Baseline & 1 month & $\mathrm{t}$ & P-value \\
\hline \multirow[t]{3}{*}{ Weight (kg) } & Control & $128.46 \pm 15.87$ & $123.86 \pm 14.07$ & -1.779 & 0.084 \\
\hline & 10DF & $132.82 \pm 17.29$ & $127.93 \pm 15.89$ & -1.968 & 0.056 \\
\hline & $20 \mathrm{DF}$ & $130.75 \pm 15.53$ & $126.00 \pm 15.85^{\mathrm{a}}$ & -2.232 & 0.031 \\
\hline \multirow[t]{3}{*}{ BMI $\left(\mathrm{kg} / \mathrm{m}^{2}\right)$} & Control & $75.54 \pm 10.72$ & $76.05 \pm 9.32$ & 0.375 & 0.710 \\
\hline & 10DF & $79.58 \pm 12.2$ & $76.40 \pm 8.03$ & -1.894 & 0.066 \\
\hline & $20 \mathrm{DF}$ & $79.15 \pm 10.23$ & $78.38 \pm 8.39$ & -0.517 & 0.608 \\
\hline \multirow[t]{3}{*}{ Waist circumference $(\mathrm{cm})$} & Control & $69.57 \pm 8.48$ & $67.27 \pm 8.29^{\mathrm{b}}$ & -2.841 & 0.007 \\
\hline & 10DF & $65.26 \pm 10.20$ & $63.50 \pm 9.56^{\mathrm{a}}$ & -2.656 & 0.012 \\
\hline & 20DF & $69.49 \pm 12.99$ & $68.03 \pm 12.52$ & -1.325 & 0.193 \\
\hline \multirow[t]{3}{*}{ Hip circumference $(\mathrm{cm})$} & Control & $25.65 \pm 2.62$ & $24.83 \pm 2.83^{\mathrm{a}}$ & -2.715 & 0.010 \\
\hline & 10DF & $24.80 \pm 2.92$ & $24.16 \pm 2.82^{\mathrm{a}}$ & -2.535 & 0.015 \\
\hline & $20 \mathrm{DF}$ & $25.33 \pm 3.96$ & $24.75 \pm 3.42$ & -1.343 & 0.187 \\
\hline \multirow[t]{3}{*}{ Waist/hip ratio } & Control & $90.51 \pm 8.05$ & $89.95 \pm 7.29$ & -0.677 & 0.502 \\
\hline & $10 \mathrm{DF}$ & $90.71 \pm 9.81$ & $88.18 \pm 8.24^{b}$ & -2.822 & 0.007 \\
\hline & 20DF & $91.31 \pm 10.96$ & $91.03 \pm 9.41$ & 0.088 & 0.930 \\
\hline \multirow[t]{3}{*}{ Systolic pressure (mmHg) } & Control & $100.95 \pm 7.69$ & $99.08 \pm 6.86^{\mathrm{a}}$ & -2.373 & 0.023 \\
\hline & 10DF & $100.06 \pm 7.05$ & $97.60 \pm 7.10^{\mathrm{b}}$ & -3.199 & 0.003 \\
\hline & 20DF & $100.58 \pm 7.04$ & $100.24 \pm 7.14$ & -0.255 & 0.800 \\
\hline \multirow[t]{3}{*}{ Diastolic pressure (mmHg) } & Control & $0.90 \pm 0.06$ & $0.91 \pm 0.05$ & 1.211 & 0.234 \\
\hline & 10DF & $0.91 \pm 0.06$ & $0.90 \pm 0.06$ & -0.240 & 0.812 \\
\hline & $20 \mathrm{DF}$ & $0.91 \pm 0.06$ & $0.91 \pm 0.06$ & 0.448 & 0.657 \\
\hline \multirow[t]{3}{*}{ Fasting blood glucose $(\mathrm{mmol} / \mathrm{l})$} & Control & $8.12 \pm 2.78$ & $7.67 \pm 2.87$ & -1.162 & 0.253 \\
\hline & 10DF & $8.26 \pm 2.84$ & $7.26 \pm 2.94^{\mathrm{a}}$ & -2.684 & 0.011 \\
\hline & 20DF & $8.71 \pm 2.91$ & $6.13 \pm 1.18^{b}$ & -5.957 & $<0.001$ \\
\hline \multirow[t]{3}{*}{ 2-h blood glucose $(\mathrm{mmol} / \mathrm{l})$} & Control & $12.55 \pm 4.39$ & $11.27 \pm 4.75^{\mathrm{a}}$ & -2.667 & 0.011 \\
\hline & 10DF & $12.50 \pm 4.20$ & $9.73 \pm 3.81^{\mathrm{b}}$ & -4.409 & $<0.001$ \\
\hline & 20DF & $12.54 \pm 3.88$ & $8.08 \pm 2.77^{\mathrm{b}}$ & -7.721 & $<0.001$ \\
\hline \multirow[t]{3}{*}{ Fasting insulin (mU/l) } & Control & $9.55 \pm 7.06$ & $7.43 \pm 5.23^{\mathrm{a}}$ & -2.729 & 0.010 \\
\hline & 10DF & $9.27 \pm 5.75$ & $7.24 \pm 4.72^{\mathrm{a}}$ & -2.371 & 0.023 \\
\hline & 20DF & $9.81 \pm 5.60$ & $7.77 \pm 4.17^{\mathrm{b}}$ & -2.819 & 0.008 \\
\hline \multirow[t]{3}{*}{ 2-h insulin (mU/l) } & Control & $23.78 \pm 16.52$ & $20.72 \pm 15.92^{\mathrm{a}}$ & -2.446 & 0.019 \\
\hline & 10DF & $24.94 \pm 23.20$ & $19.72 \pm 11.02^{\mathrm{b}}$ & -3.435 & 0.001 \\
\hline & $20 \mathrm{DF}$ & $24.96 \pm 14.72$ & $21.37 \pm 11.86^{\mathrm{b}}$ & -4.704 & $<0.001$ \\
\hline \multirow[t]{3}{*}{ HOMA-IR } & Control & $3.34 \pm 2.64$ & $2.56 \pm 2.17^{\mathrm{b}}$ & -3.794 & 0.001 \\
\hline & $10 \mathrm{DF}$ & $3.40 \pm 2.59$ & $2.30 \pm 1.58^{b}$ & -3.182 & 0.003 \\
\hline & 20DF & $3.92 \pm 2.77$ & $2.12 \pm 1.29^{\mathrm{b}}$ & -4.678 & $<0.001$ \\
\hline \multirow[t]{3}{*}{ Fasting C-peptide (ng/ml) } & Control & $2.25 \pm 1.03$ & $1.94 \pm 0.96$ & -1.856 & 0.072 \\
\hline & 10DF & $2.78 \pm 1.57$ & $1.93 \pm 0.79^{b}$ & -3.893 & $<0.001$ \\
\hline & 20DF & $2.59 \pm 1.39$ & $1.96 \pm 0.78^{b}$ & -3.212 & 0.003 \\
\hline \multirow[t]{3}{*}{ 2-h C-peptide (ng/ml) } & Control & $5.28 \pm 4.05$ & $4.28 \pm 2.65$ & -2.008 & 0.052 \\
\hline & 10DF & $6.57 \pm 4.20$ & $3.59 \pm 1.78^{b}$ & -2.979 & 0.005 \\
\hline & 20DF & $6.15 \pm 3.01$ & $3.27 \pm 2.03^{\mathrm{b}}$ & -1.869 & 0.004 \\
\hline \multirow[t]{3}{*}{ Glycated albumin (\%) } & Control & $18.84 \pm 4.74$ & $17.61 \pm 4.35$ & -1.859 & 0.071 \\
\hline & 10DF & $20.00 \pm 4.31$ & $15.27 \pm 3.81^{b}$ & -7.361 & $<0.001$ \\
\hline & 20DF & $20.64 \pm 5.35$ & $12.61 \pm 1.92^{\mathrm{b}}$ & -8.948 & $<0.001$ \\
\hline \multirow[t]{3}{*}{ Cholesterol (mmol/l) } & Control & $4.83 \pm 2.16$ & $4.81 \pm 1.01$ & -0.039 & 0.969 \\
\hline & 10DF & $4.84 \pm 1.17$ & $4.93 \pm 1.02$ & 0.801 & 0.428 \\
\hline & 20DF & $5.07 \pm 1.31$ & $4.97 \pm 1.10$ & -0.463 & 0.646 \\
\hline \multirow[t]{3}{*}{ Triglyceride (mmol/l) } & Control & $2.08 \pm 1.58$ & $2.45 \pm 2.80$ & 1.019 & 0.315 \\
\hline & 10DF & $2.68 \pm 2.68$ & $1.77 \pm 1.96^{\mathrm{b}}$ & -2.891 & 0.006 \\
\hline & 20DF & $2.40 \pm 2.68$ & $1.55 \pm 1.21^{\mathrm{b}}$ & -3.177 & 0.003 \\
\hline \multirow[t]{3}{*}{$\mathrm{HDL}(\mathrm{mmol} / \mathrm{l})$} & Control & $1.12 \pm 0.42$ & $1.02 \pm 0.33$ & -1.457 & 0.154 \\
\hline & 10DF & $1.27 \pm 0.41$ & $1.18 \pm 0.40$ & -1.896 & 0.065 \\
\hline & $20 \mathrm{DF}$ & $1.36 \pm 0.71$ & $1.12 \pm 0.27^{\mathrm{a}}$ & -2.359 & 0.023 \\
\hline
\end{tabular}


Table III. Continued.

\begin{tabular}{|c|c|c|c|c|c|}
\hline Parameter & Group & Baseline & 1 month & $\mathrm{t}$ & P-value \\
\hline \multirow[t]{3}{*}{ LDL (mmol/l) } & Control & $2.92 \pm 2.71$ & $2.76 \pm 1.62$ & -0.452 & 0.654 \\
\hline & 10DF & $2.45 \pm 0.74$ & $2.32 \pm 0.61$ & -1.348 & 0.185 \\
\hline & $20 \mathrm{DF}$ & $2.90 \pm 0.80$ & $2.31 \pm 0.76^{b}$ & -4.683 & $<0.001$ \\
\hline \multirow[t]{3}{*}{ Lpa (mg/l) } & Control & $292.16 \pm 258.47$ & $378.35 \pm 315.53^{\mathrm{b}}$ & 4.210 & $<0.001$ \\
\hline & 10DF & $311.38 \pm 282.61$ & $371.38 \pm 207.30^{\mathrm{a}}$ & 2.581 & 0.014 \\
\hline & $20 \mathrm{DF}$ & $313.15 \pm 227.88$ & $362.60 \pm 186.65^{b}$ & 3.126 & 0.003 \\
\hline \multirow[t]{3}{*}{$\operatorname{ApoA}(\mathrm{g} / \mathrm{l})$} & Control & $1.12 \pm 0.27$ & $1.04 \pm 0.17$ & -2.013 & 0.052 \\
\hline & 10DF & $1.25 \pm 0.29$ & $1.19 \pm 0.26$ & -1.315 & 0.196 \\
\hline & $20 \mathrm{DF}$ & $1.29 \pm 0.49$ & $1.20 \pm 0.23$ & -1.165 & 0.251 \\
\hline \multirow[t]{3}{*}{ ApoB (g/l) } & Control & $1.02 \pm 0.36$ & $0.95 \pm 0.42$ & -1.842 & 0.074 \\
\hline & 10DF & $1.30 \pm 0.49$ & $1.16 \pm 0.59$ & -1.572 & 0.124 \\
\hline & $20 \mathrm{DF}$ & $1.52 \pm 0.48$ & $1.18 \pm 0.35^{\mathrm{b}}$ & -3.627 & 0.001 \\
\hline
\end{tabular}

Data are presented as the mean \pm standard deviation. Significant $\mathrm{P}$-values $(\mathrm{P}<0.05)$ are indicated in bold. ${ }^{\mathrm{a}} \mathrm{P}<0.05$ and ${ }^{\mathrm{b}} \mathrm{P}<0.01 \mathrm{vs}$. the baseline 10DF, $10 \mathrm{~g}$ /day dietary fiber; 20DF, $20 \mathrm{~g}$ /day dietary fiber; BMI, body mass index; HOMA-IR, homeostatic model assessment-insulin resistance; HDL, high-density lipoprotein; LDL, low-density lipoprotein; Lpa, lipoprotein(a); Apo, apolipoprotein.

Table IV. Comparison of the statistical differences between the pre- and post-treatment measurements of various parameters among the three groups.

\begin{tabular}{|c|c|c|c|c|c|c|c|}
\hline Parameter & Control & $10 \mathrm{DF}$ & $20 \mathrm{DF}$ & $\mathrm{P} 1$ & $\mathrm{P} 2$ & P3 & $\mathrm{P} 4$ \\
\hline Weight (kg) & $-2.30 \pm 4.92$ & $-1.76 \pm 4.20$ & $-1.47 \pm 7.02$ & 0.659 & 0.865 & 0.496 & 0.391 \\
\hline BMI $\left(\mathrm{kg} / \mathrm{m}^{2}\right)$ & $-0.82 \pm 1.84$ & $-0.65 \pm 1.62$ & $-0.58 \pm 2.72$ & 0.877 & 0.901 & 0.720 & 0.622 \\
\hline Waist circumference $(\mathrm{cm})$ & $-0.57 \pm 5.10$ & $-2.54 \pm 5.69$ & $0.09 \pm 6.44^{\mathrm{a}}$ & 0.051 & 0.095 & 0.493 & 0.018 \\
\hline Hip circumference $(\mathrm{cm})$ & $-1.86 \pm 4.78$ & $-2.46 \pm 4.87$ & $-0.21 \pm 5.09^{\mathrm{a}}$ & 0.074 & 0.428 & 0.150 & 0.025 \\
\hline Waist/hip ratio & $0.01 \pm 0.05$ & $0.00 \pm 0.05$ & $0.00 \pm 0.06$ & 0.738 & 0.444 & 0.781 & 0.625 \\
\hline Systolic pressure (mmHg) & $-4.59 \pm 15.71$ & $-4.93 \pm 15.83$ & $-4.75 \pm 13.46$ & 0.833 & 0.546 & 0.747 & 0.772 \\
\hline Diastolic pressure (mmHg) & $-0.51 \pm 8.34$ & $-3.18 \pm 10.60$ & $-0.78 \pm 9.48$ & 0.399 & 0.405 & 0.639 & 0.181 \\
\hline Fasting blood glucose (mmol/l) & $-0.45 \pm 2.36$ & $-1.00 \pm 2.36$ & $-2.58 \pm 2.74^{\mathbf{b}, \mathbf{c}}$ & $<0.001$ & 0.303 & $<0.001$ & 0.004 \\
\hline $2 \mathrm{~h}$ blood glucose $(\mathrm{mmol} / \mathrm{l})$ & $-1.29 \pm 2.94$ & $-2.77 \pm 3.97^{\mathrm{d}}$ & $-4.46 \pm 3.65^{\mathbf{a}, \mathbf{b}}$ & $<0.001$ & 0.033 & $<0.001$ & 0.016 \\
\hline Fasting insulin (mU/l) & $-2.11 \pm 4.71$ & $-2.04 \pm 5.43$ & $-2.04 \pm 4.58$ & 0.941 & 0.937 & 0.803 & 0.738 \\
\hline $2 \mathrm{~h}$ insulin $(\mathrm{mU} / \mathrm{l})$ & $-3.07 \pm 7.62$ & $-5.22 \pm 9.61$ & $-4.69 \pm 6.31$ & 0.514 & 0.250 & 0.519 & 0.605 \\
\hline HOMA-IR & $-0.79 \pm 1.26$ & $-1.11 \pm 2.20$ & $-1.80 \pm 2.44^{\mathrm{d}}$ & 0.081 & 0.344 & 0.026 & 0.186 \\
\hline Fasting C-peptide (ng/ml) & $-0.32 \pm 1.05$ & $-0.85 \pm 1.37$ & $-0.62 \pm 1.23$ & 0.684 & 0.386 & 0.690 & 0.627 \\
\hline 2 h C-peptide (ng/ml) & $-1.00 \pm 3.04$ & $-1.99 \pm 4.22$ & $-0.88 \pm 2.97$ & 0.127 & 0.989 & 0.085 & 0.076 \\
\hline Glycated albumin (\%) & $-1.23 \pm 4.03$ & $-4.73 \pm 4.06$ & $-8.75 \pm 5.39^{\mathbf{b}, \mathbf{c}}$ & $<0.001$ & $<0.001$ & $<0.001$ & $<0.001$ \\
\hline Cholesterol (mmol/l) & $-0.01 \pm 1.80$ & $0.10 \pm 0.76$ & $-0.93 \pm 1.32$ & 0.848 & 0.570 & 0.723 & 0.828 \\
\hline Triglyceride (mmol/l) & $0.37 \pm 2.19$ & $-0.91 \pm 1.98$ & $-0.84 \pm 1.69^{b}$ & 0.008 & 0.008 & 0.005 & 0.861 \\
\hline $\mathrm{HDL}(\mathrm{mmol} / \mathrm{l})$ & $0.10 \pm 0.41$ & $0.09 \pm 0.30$ & $0.24 \pm 0.65$ & 0.251 & 0.818 & 0.129 & 0.187 \\
\hline $\mathrm{LDL}(\mathrm{mmol} / \mathrm{l})$ & $-0.16 \pm 2.15$ & $-0.13 \pm 0.61$ & $-0.58 \pm 0.79^{\mathrm{d}}$ & 0.091 & 0.195 & 0.030 & 0.368 \\
\hline Lpa (mg/l) & $-86.19 \pm 124.52$ & $-60.00 \pm 147.05$ & $-49.45 \pm 100.06$ & 0.436 & 0.368 & 0.210 & 0.717 \\
\hline $\operatorname{ApoA}(g / 1)$ & $-0.07 \pm 0.22$ & $-0.06 \pm 0.30^{\mathrm{d}}$ & $-0.09 \pm 0.47^{\mathrm{d}}$ & 0.028 & 0.021 & 0.018 & 0.922 \\
\hline ApoB (g/l) & $-0.07 \pm 0.24$ & $-0.14 \pm 0.57$ & $-0.34 \pm 0.59$ & 0.609 & 0.344 & 0.776 & 0.506 \\
\hline Urea nitrogen (mmol/l) & $-0.60 \pm 3.34$ & $-0.41 \pm 1.75$ & $-0.41 \pm 1.79$ & 0.921 & 0.692 & 0.781 & 0.904 \\
\hline Creatinine $(\mu \mathrm{mol} / \mathrm{l})$ & $-1.94 \pm 17.60$ & $2.32 \pm 10.34$ & $1.64 \pm 11.07$ & 0.527 & 0.291 & 0.371 & 0.868 \\
\hline Uric acid $(\mu \mathrm{mol} / \mathrm{l})$ & $3.92 \pm 77.67$ & $-10.70 \pm 65.16$ & $1.43 \pm 79.87$ & 0.802 & 0.599 & 0.943 & 0.541 \\
\hline
\end{tabular}

Data are presented as the mean \pm standard deviation. Significant $\mathrm{P}$-values $(\mathrm{P}<0.05)$ are indicated in bold. ${ }^{\mathrm{P}}<0.05 \mathrm{vs}$. the $10 \mathrm{DF}$ group; ${ }^{\mathrm{b}} \mathrm{P}<0.01 \mathrm{vs}$. the control group; ${ }^{\mathrm{C}} \mathrm{P}<0.01 \mathrm{vs}$. the $10 \mathrm{DF}$ group; ${ }^{\mathrm{d}} \mathrm{P}<0.05$ vs. the control group. $\mathrm{P} 1$, analysis of variance among the three groups; $\mathrm{P} 2$, comparison between the 10DF and control groups; P3, comparison between the 20DF and control groups; P4, comparison between the 10DF and 20DF groups; 10DF, 10 g/day dietary fiber; 20DG, 20 g/day dietary fiber; BMI, body mass index; HOMA-IR, homeostatic model assessment-insulin resistance; HDL, high-density lipoprotein; LDL, low-density lipoprotein; Lpa, lipoprotein(a); Apo, apolipoprotein. 
Table V. Renal function data.

\begin{tabular}{lcccrr}
\hline Parameter & Group & Baseline & 1 month & $\mathrm{t}$ & P-value \\
\hline Urea nitrogen $(\mathrm{mmol} / \mathrm{l})$ & Control & $6.00 \pm 3.68$ & $5.40 \pm 1.49$ & -1.093 & 0.282 \\
& $10 \mathrm{DF}$ & $5.91 \pm 1.73$ & $5.50 \pm 1.44$ & -1.468 & 0.150 \\
& $20 \mathrm{DF}$ & $5.86 \pm 2.01$ & $5.45 \pm 1.53$ & -1.438 & 0.158 \\
Creatinine $(\mu \mathrm{mol} / \mathrm{l})$ & Control & $64.09 \pm 22.49$ & $62.15 \pm 18.73$ & -0.670 & 0.507 \\
& $10 \mathrm{DF}$ & $59.58 \pm 20.60$ & $61.91 \pm 19.99$ & 1.421 & 0.163 \\
& $20 \mathrm{DF}$ & $60.36 \pm 15.28$ & $62.00 \pm 15.42$ & 0.937 & 0.354 \\
Uric acid $(\mu \mathrm{mol} / \mathrm{l})$ & Control & $289.65 \pm 104.52$ & $293.57 \pm 88.28$ & 0.307 & 0.761 \\
& $10 \mathrm{DF}$ & $304.83 \pm 92.57$ & $294.13 \pm 71.33$ & -1.039 & 0.305 \\
& $20 \mathrm{DF}$ & $296.77 \pm 76.57$ & $298.20 \pm 67.34$ & 0.113 & 0.911 \\
\hline
\end{tabular}

Data are presented as the mean \pm standard deviation. $10 \mathrm{DF}, 10 \mathrm{~g} /$ day dietary fiber; 20DF, $20 \mathrm{~g} /$ day dietary fiber.

Table VI. Defecation analysis.

\begin{tabular}{|c|c|c|c|c|c|c|}
\hline \multirow[b]{2}{*}{ Group } & \multicolumn{2}{|c|}{ 7-day defecation frequency } & \multicolumn{2}{|c|}{ Defecation sensation score } & \multicolumn{2}{|c|}{ Stool characteristics score } \\
\hline & Baseline & 1 month & Baseline & 1 month & Baseline & 1 month \\
\hline Control & $5.27 \pm 2.33$ & $5.27 \pm 2.16$ & $1.22 \pm 1.10$ & $1.22 \pm 1.08$ & $1.49 \pm 1.04$ & $1.45 \pm 1.04$ \\
\hline $10 \mathrm{DF}$ & $5.40 \pm 2.35$ & $6.33 \pm 1.00^{\mathrm{a}, \mathrm{b}}$ & $1.35 \pm 1.60$ & $0.50 \pm 0.60^{\mathrm{a}, \mathrm{b}}$ & $1.45 \pm 1.04$ & $0.65 \pm 0.77^{\mathrm{a}, \mathrm{b}}$ \\
\hline $20 \mathrm{DF}$ & $5.25 \pm 2.42$ & $6.45 \pm 1.22^{\mathrm{a}, \mathrm{b}}$ & $1.18 \pm 1.04$ & $0.55 \pm 0.64^{\mathrm{a}, \mathrm{b}}$ & $1.48 \pm 1.01$ & $0.63 \pm 0.68^{a, b}$ \\
\hline P-value & 0.954 & $0.002^{\mathrm{c}}$ & 0.752 & $<0.001^{\mathrm{c}}$ & 0.987 & $<0.001^{\mathrm{c}}$ \\
\hline
\end{tabular}

Data are presented as the mean \pm standard deviation. ${ }^{\mathrm{a}} \mathrm{P}<0.05$ vs. the control group; ${ }^{\mathrm{b}} \mathrm{P}<0.05$ vs. the baseline; ${ }^{\mathrm{c}} \mathrm{P}<0.05$; analysis of variance among the three groups. 10DF, $10 \mathrm{~g}$ /day dietary fiber; 20DF, $20 \mathrm{~g}$ /day dietary fiber.

patients in the 10DF group complained of excessive flatulence. However, increased soluble DF intake significantly improved defecation frequency, defecation ease and stool characteristics $(\mathrm{P}<0.05$; Table VI).

\section{Discussion}

Dietary factors have been demonstrated to impact the development of DM2 and its associated complications (15). A survey conducted in the US reported an average DF intake of $17 \mathrm{~g} /$ day in non-diabetic individuals, with an average of $16 \mathrm{~g} /$ day demonstrated in diabetic patients (16). In the present study, patients consumed an average of $15.9 \mathrm{~g} /$ day DF, which was lower than that recommended by the ADA and may be partially due to ignorance regarding the beneficial effects of DF on glycemic control. Therefore, the present study aimed to investigate the effects of increased DF intake on glycemic control and the underlying mechanisms.

In the present study, 2-h blood glucose, fasting insulin and Lpa levels, and the insulin resistance index, were significantly improved from baseline in all three groups, thus suggesting that MNT treatment was able to significantly impact blood glucose levels and glycemic control. In three large randomized trials, including the China Da Qing Diabetes Prevention study (17), the Finnish Diabetes Prevention Study (18) and the Diabetes Prevention Program trial (19), it was demonstrated that the progression from impaired glucose tolerance to DM2 may be delayed or even prevented by diet and exercise. Furthermore, dietary interventions aimed at improving diet quality have been shown to be effective for controlling DM2 (2), which is consistent with the results of the present study.

Increasing DF intake, which is one of the goals of nutritional counseling, deserves greater attention due to its ability to reduce total cholesterol levels and hyperglycemia in patients with impaired glucose tolerance and DM2 (20). In addition, increased fiber intake was shown to improve insulin sensitivity and reduce systemic inflammation $(21,22)$. Previous studies have demonstrated that high-fiber diets (30 g/day) altered biochemical parameters, reduced the severity of DM2 and decreased the occurrence of risk factors associated with cardiovascular disease $(21,23)$. According to Weickert et al (21), nutritional educational studies involving dietary restrictions are typically met with poor treatment compliance. Participants in a previous study were encouraged to progressively alter their eating behaviors, including increasing the frequency of meals and increasing the intake of complex carbohydrates, DF, fruits, and vegetables, as well as polyunsaturated and monounsaturated fatty acids, including fish and olive oils, respectively (21). However, MNT treatment was unable to increase DF intake in the subjects (21). Similarly, another interventional study involving nutritional education has failed to increase fiber intake (24). In the present 
study, patients received extra soluble DF and were required to recount the remaining soluble DF during a phone consultation every week. Soluble DF has been associated with lower postprandial glucose levels and increased insulin sensitivity in diabetic and healthy subjects; these effects were generally attributed to the viscous and/or gelling properties of soluble fiber (25). Soluble DF exerts physiological effects on the stomach and small intestine that modulate postprandial glycemic responses, including delaying gastric emptying (26), which accounts for $\sim 35 \%$ of the variance in peak glucose concentrations following the ingestion of oral glucose (27), modulating gastrointestinal myoelectrical activity and delaying small bowel transit $(28,29)$, reducing glucose diffusion through the unstirred water layer (30), and reducing the accessibility of $\alpha$-amylase to its substrates due to the increased viscosity of gut contents (31). Notably, the increased viscosity and gel-forming properties of soluble fiber are predominantly responsible for its glycemic effect, since the hypoglycemic effect can be reversed by the hydrolysis of guar gum or following ultra-high heating and homogenization (26). In addition, the intestinal absorption of carbohydrates was prolonged by soluble DF, which was partially due to altered incretin levels, including increased glucagon-like peptide 1 levels (32). In experimental clamp studies, soluble DF also influenced peripheral glucose uptake mechanisms $(33,34)$, including increasing skeletal muscle expression of the insulin-responsive glucose transporter type 4 (GLUT-4), which enhances skeletal muscle uptake, augments insulin sensitivity and normalizes blood glucose (34). In humans, various fatty acids stimulate the expression of peroxisome proliferator-activated receptor- $\gamma$, which increases adipocyte GLUT-4 levels (35).

Measuring the levels of glycated proteins, including hemoglobin A1c (HbAlc), GA and fructosamine, is the most reliable method for assessing long-term glycemic control in diabetic patients (36). Since glycation may occur throughout the lifespan of hemoglobin and serum proteins, the level of glycated proteins is able to reflect the degree of hyperglycemia during the lifespan of these factors (37). HbAlc, which is the most widely used marker, can be used to quantify the amount of circulating hemoglobin that has chemically reacted with glucose, and reflects ambient blood glucose levels over the previous 120 days and the preceding 30 days $(38,39)$. Of the various glycated proteins, serum GA has been identified as a useful and rapid indicator of glycemic control for diabetic patients, as the turnover of serum albumin is markedly shorter, with a half-life of 17 days, as compared with that of HbAlc (40). Circulating albumin is strongly glycated on lysine 4 residues, and the glycation reaction occurs 10 times more rapidly than the glycation of hemoglobin (41). Therefore, it is likely that glycemic fluctuations and excursions influence the glycation of albumin more directly than hemoglobin. Inaba et al (42) have previously reported that HbA1c quantification underestimates the long-term glycemic control in dialysis patients with diabetes after comparing the mean of random blood glucose concentrations, HbAlc, and the percentage of GA (\%GA). In addition, it was demonstrated that the \%GA assay provided a more accurate assessment of glycemic control among Japanese hemodialysis patients. The present study detected GA levels in patients, since the intervention period was only one month.
$\mathrm{C}$-peptide is a cleavage product of proinsulin that is secreted by pancreatic $\beta$-cells in equimolar amounts together with insulin (43). Although a considerable amount of insulin is extracted by the liver, C-peptide is subject to negligible first-pass metabolism by the liver, thereby permitting its use as a surrogate marker for endogenous insulin secretion (44). C-peptide is thought to be an inert by-product of insulin synthesis and has been of great value for elucidating the pathophysiology of type 1 and type 2 diabetes mellitus (45). Notably, C-peptide levels have previously been used as a biomarker of $\beta$-cell function (46). The present study demonstrated that, although soluble DF was able to significantly improve fasting blood glucose, 2-h blood glucose and GA levels, and the insulin resistance index, it was unable to improve the levels of fasting insulin, 2-h insulin, fasting C-peptide and 2-h C-peptide, thus suggesting that soluble DF does not affect the secretory function of the islets of Langerhans when used for a short duration. The reason for this remains unclear and will be the key focus of our follow-up studies.

In order to confirm the safety of increased soluble DF intake, the renal functions of all patients were assessed and routine blood tests and urinalyses were performed (data not shown). No significant adverse reactions were found, with the exception of increased flatulence in some patients. Furthermore, the results suggested that soluble DF was able to significantly improve constipation and diarrhea in patients with D2M and improve defecation ease and stool characteristics. Notably, there were no significant differences in these effects between the 10 and $20 \mathrm{~g} /$ day DF groups. Previous studies have suggested that the effects of DF on metabolic and cardiovascular outcomes are associated with gastrointestinal function, which may be reasonable considering the demonstrated links between DF and satiation $(47,48)$.

The present study demonstrated that high doses of DF were able to improve numerous metabolic indicators in patients with DM2; however, further research is required to determine the specific mechanisms underlying the effects of DF. Such findings may have a large impact on the prevention and clinical treatment of DM2.

In conclusion, the present study demonstrated that MNT was able to improve 2-h blood glucose and fasting insulin levels, and the insulin resistance index, and was effective at maintaining glycemic control. To the best of our knowledge, the present study is the first to demonstrate that increased intake of soluble DF over a short-duration in Chinese patients with D2M was able to significantly improve blood glucose levels and insulin resistance without affecting the secretory functions of the islets of Langerhans. Therefore, the authors of the present study recommend that dietary guidelines for patients with D2M should stress the importance of increased DF intake.

\section{Acknowledgements}

The present study was supported by the National Science and Technology Support Program (grant no. 2009BAI80B04), the Chongqing Science and Technology Commission (grant no. CSTC, 2011AB5040), 'the 11th Five-year Plan' for the National Key Technology Research and Development Program (grant no. 2008BAI58B06), and the Innovation 
Project of Chongqing Key Laboratory of Nutrition and Food Safety (grant no. 2006CA1003).

\section{Referen5ces}

1. Kramer CK, Leitão CB, Pinto LC, Boza J, Silveiro SP, Gross JL and Canani LH: Risk factors for micro and macrovascular disease in black and white patients with type 2 diabetes mellitus. Rev Assoc Med Bras 55: 308-314, 2009.

2. Glauber $\mathrm{H}$ and Karnieli E: Preventing type 2 diabetes mellitus: A call for personalized intervention. Perm J 17: 74-79, 2013.

3. Pimentel GD, Arimura ST, de Moura BM, Silva ME and de Sousa MV: Short-term nutritional counseling reduces body mass index, waist circumference, triceps skinfold and triglycerides in women with metabolic syndrome. Diabetol Metab Syndr 2: 13 , 2010.

4. American Diabetes Association: Nutrition recommendations and principles for people with diabetes mellitus. Diabetes Care 23 (Suppl 1): S43-S46, 2000.

5. National Cholesterol Education Program (NCEP) Expert Panel on Detection, Evaluation and Treatment of High Blood Cholesterol in Adults (Adult Treatment Panel III): Third report of the national cholesterol education program (ncep) expert panel on detection, evaluation, and treatment of high blood cholesterol in Adults (Adult Treatment Panel III) final report. Circulation 106: 3143-3421, 2002.

6. Ortega RM, Palencia A and López-Sobaler AM: Improvement of cholesterol levels and reduction of cardiovascular risk via the consumption of phytosterols. Br J Nutr 96 (Suppl 1): S89-S93, 2006.

7. Theuwissen E and Mensink RP: Water-soluble dietary fibers and cardiovascular disease. Physiol Behav 94: 285-292, 2008.

8. Bernaud FS and Rodrigues TC: Dietary fiber-adequate intake and effects on metabolism health. Arq Bras Endocrinol Metabol 57: 397-405, 2013 (In Portuguese).

9. Frankenfeld CL: Cardiometabolic risk factors are associated with high urinary enterolactone concentration, independent of urinary enterodiol concentration and dietary fiber intake in adults. J Nutr 144: 1445-1453, 2014.

10. Breneman CB and Tucker L: Dietary fibre consumption and insulin resistance-the role of body fat and physical activity. Br J Nutr 110: 375-383, 2013.

11. Bito H, Hamaguchi N, Hirai H and Ogawa K: Safety evaluation of a newly-developed dietary fiber: Resistant glucan mixture. J Toxicol Sci 41: 33-44, 2016

12. Enes P, Martín-Frías M, Álvarez MÁ, Yelmo R, Alonso M and Barrio R: Achievement of metabolic control goals set by the American diabetes association and the international society for pediatric and adolescent diabetes in pediatric patients with type 1 diabetes from Spain. Diabetes Res Clin Pract 107: 300-305, 2015

13. Rao SS, Kavlock R and Rao S: Influence of body position and stoo characteristics on defecation in humans. Am J Gastroenterol 101: 2790-2796, 2006.

14. Hill NR, Levy JC and Matthews DR: Expansion of the homeostasis model assessment of $\beta$-cell function and insulin resistance to enable clinical trial outcome modeling through the interactive adjustment of physiology and treatment effects: iHOMA2. Diabetes Care 36 2324-2330, 2013.

15. Lapik IA, Sokol'nikov AA, Sharafetdinov KhKh, Sentsova TB and Plotnikova OA: Assessment of efficiency of dietotherapy with addition of a vitamin-mineral complex in patients with diabetes mellitus type 2. Vopr Pitan 83: 74-81, 2014 (In Russian).

16. Gunter EW, Lewis BG and Koncikowski SM: Laboratory procedures used for the third national health and nutrition examination survey (NHANES III), 1988-1994. Atlanta, GA National center for environmental health, center for disease control and prevention, 1966. Available at: http://www.cdc. gov/nchs/data/nhanes/nhanes3/cdrom/nchs/manuals/labman.pdf (accessed October 27, 2014).

17. Li G, Zhang P, Wang J, Gregg EW, Yang W, Gong Q, Li H, Li H, Jiang Y, An Y, Shuai Y, Zhang B, Zhang J, Thompson TJ, Gerzoff RB, Roglic G, Hu Y, Bennett PH: The long-term effect of lifestyle interventions to prevent diabetes in the China Da Qing Diabetes Prevention Study: A 20-year follow-up study. The Lancet 371: 1783-1789, 2008.

18. Tuomilehto J, Lindström J, Eriksson JG, Valle TT, Hämäläinen H, Ilanne-Parikka P, Keinänen-Kiukaanniemi P, Laakso M, Louheranta A, Rastas M, et al; Finnish Diabetes Prevention Study Group: Prevention of type 2 diabetes mellitus by changes in lifestyle among subjects with impaired glucose tolerance. N Engl J Med 344: 1343-1350, 2001
19. Knowler WC, Barrett-Connor E, Fowler SE, Hamman RF, Lachin JM, Walker EA and Nathan DM; Diabetes Prevention Program Research Group: Reduction in the incidence of type 2 diabetes with lifestyle intervention or metformin. N Engl J Med 346: 393-403, 2002.

20. Behall KM, Scholfield DJ, Hallfrisch JG and Liljeberg-Elmstãhl HG: Consumption of both resistant starch and beta-glucan improves postprandial plasma glucose and insulin in women. Diabetes Care 29: 976-981, 2006.

21. Weickert MO, Möhlig M, Schöel C, Arafat AM, Otto B, Viehoff H, Koebnick C, Kohl A, Spranger J and Pfeiffer AF: Cereal fiber improves whole-body insulin sensitivity in overweight and obese women. Diabetes Care 29: 775-780, 2006.

22. Qi L, Van Dam RM, Liu S, Franz M, Mantzoros C and Hu FB: Wholegrain, bran, and cereal fiber intakes and markers of systemic inflammation in diabetic women. Diabetes Care 29: 207-211, 2006.

23. American Diabetes Association: Evidence-based nutrition principles and recommendations for the treatment and prevention of diabetes and related complications. Diabetes Care 25: 202-212, 2002.

24. Bihan H, Takbou K, Cohen R, Michault A, Boitou F, Reach G and Le Clésiau H: Impact of short-duration lifestyle intervention in collaboration with general practitioners in patients with the metabolic syndrome. Diabetes Metab 35: 185-191, 2009.

25. Sierra M, García JJ, Fernández N, Diez MJ and Calle AP: Therapeutic effects of psyllium in type 2 diabetic patients. Eur J Clin Nutr 56: 830-842, 2002.

26. Jenkins DJ, Wolever TM, Leeds AR, Gassull MA, Haisman P, Dilawari J, Goff DV, Metz GL and Alberti KG: Dietary fibres, fibre analogues, and glucose tolerance: Importance of viscosity. Br Med J 1: 1392-1394, 1978.

27. Jones KL, Horowitz M, Carney BI, Wishart JM, Guha S and Green L: Gastric emptying in early noninsulin-dependent diabetes mellitus. J Nucl Med 37: 1643-1648, 1996.

28. Cherbut C, Albina E, Champ M, Doublier JL and Lecannu G: Action of guar gums on the viscosity of digestive contents and on the gastrointestinal motor function in pigs. Digestion 46: 205-213, 1990

29. Schönfeld J, Evans DF and Wingate DL: Effect of viscous fiber (guar) on postprandial motor activity in human small bowel. Dig Dis Sci 42: 1613-1617, 1997.

30. Johnson IT and Gee JM: Effect of gel-forming gums on the intestinal unstirred layer and sugar transport in vitro. Gut 22: 398-403, 1981

31. Leclère CJ, Champ M, Boillot J, Guille G, Lecannu G, Molis C, Bornet F, Krempf M, Delort-Laval J and Galmiche JP: Role of viscous guar gums in lowering the glycemic response after a solid meal. Am J Clin Nutr 59: 914-921, 1994.

32. Leclère CJ, Champ M, Boillot J, Guille G, Lecannu G, Molis C, Bornet F, Krempf M, Delort-Laval J and Galmiche JP: Role of viscous guar gums in lowering the glycemic response after a solid meal. Am J Clin Nutr 59: 914-921, 1994.

33. Cameron-Smith D, Habito R, Barnett M and Collier GR: Dietary guar gum improves insulin sensitivity in streptozotocin-induced diabetic rats. J Nutr 127: 359-364, 1997.

34. Song YJ, Sawamura M, Ikeda K, Igawa S and Yamori Y: Soluble dietary fibre improves insulin sensitivity by increasing muscle GLUT-4 content in stroke-prone spontaneously hypertensive rats. Clin Exp Pharmacol Physiol 27: 41-45, 2000.

35. Park KS, Ciaraldi TP, Lindgren K, Abrams-Carter L, Mudaliar S, Nikoulina SE, Tufari SR, Veerkamp JH, Vidal-Puig A and Henry RR: Troglitazone effects on gene expression in human skeletal muscle of type II diabetes involve up-regulation of peroxisome proliferator-activated receptor-gamma. J Clin Endocrinol Metab 83: 2830-2835, 1998.

36. Rathmann W, Bongaerts B and Kostev K: Change in HbAlc after initiating second-line therapy in Type 2 diabetes: A primary care database study. Diabetes Obes Metab: Apr 7, 2016 (Epub ahead of print).

37. Tahara Y and Shima K: Kinetics of HbA1c, Glycated albumin, and fructosamine and analysis of their weight functions against preceding plasma glucose level. Diabetes Care 18: 440-447, 1995.

38. Calisti L and Tognetti S: Measure of glycosylated hemoglobin. Acta Biomed 76 (Suppl 3): S59-S62, 2005.

39. Goldstein DE, Little RR, Lorenz RA, Malone JI, Nathan D, Peterson CM and Sacks DB: Tests of glycemia in diabetes. Diabetes Care 27: 1761-1773, 2004 
40. Yoshiuchi K, Matsuhisa M, Katakami N, Nakatani Y, Sakamoto K, Matsuoka T, Umayahara Y, Kosugi K, Kaneto H, Yamasaki Y and Hori M: Glycated albumin is a better indicator for glucose excursion than glycated hemoglobin in type 1 and type 2 diabetes. Endocr J 55: 503-507, 2008.

41. Takahashi S, Uchino H, Shimizu T, Kanazawa A, Tamura Y, Sakai K, Watada H, Hirose T, Kawamori R and Tanaka Y: Comparison of glycated albumin (GA) and glycated hemoglobin (HbAlc) in type 2 diabetic patients: Usefulness of GA for evaluation of short-term changes in glycemic control. Endocr J 54 139-144, 2007.

42. Inaba M, Okuno S, Kumeda Y, Yamada S, Imanishi Y, Tabata T, Okamura M, Okada S, Yamakawa T, Ishimura E, et al: Glycated albumin is a better glycemic indicator than glycated hemoglobin values in hemodialysis patients with diabetes: Effect of anemia and erythropoietin injection. J Am Soc Nephrol 18: 896-903, 2007.
43. Horwitz DL, Kuzuya H and Rubenstein AH: Circulating serum Cpeptide. A brief review of diagnostic implications. N Engl J Med 295: 207-209, 1976.

44. Clark PM and Hales CN: How to measure plasma insulin. Diabetes Metab Rev 10: 79-90, 1994.

45. Webb PG and Bonser AM: Basal C-peptide in the discrimination of type I from type II diabetes. Diabetes Care 4: 616-619, 1981.

46. Faber OK and Binder C: C-peptide: An index of insulin secretion. Diabetes Metab Rev 2: 331-345, 1986.

47. Papathanasopoulos A and Camilleri M: Dietary fiber supplements: Effects in obesity and metabolic syndrome and relationship to gastrointestinal functions. Gastroenterology 138: 65-72, 2010.

48. Burns AM, Zitt MA, Rowe CC, Langkamp-Henken B, Mai V, Nieves C Jr, Ukhanova M, Christman MC and Dahl WJ: Diet quality improves for parents and children when almonds are incorporated into their daily diet: A randomized, crossover study. Nutr Res 36: 80-89, 2016. 